Document downloaded from:

http://hdl.handle.net/10251/38211

This paper must be cited as:

Plazas Bonilla, CE.; Gómez-Tejedor, JA.; Perilla, JE.; Gómez Ribelles, JL. (2013). Silica phase formed by sol-gel reaction in the nano- and micro-pores of a polymer hydrogel. Journal of Non-Crystalline Solids. 379:12-20. doi:10.1016/j.jnoncrysol.2013.07.018.

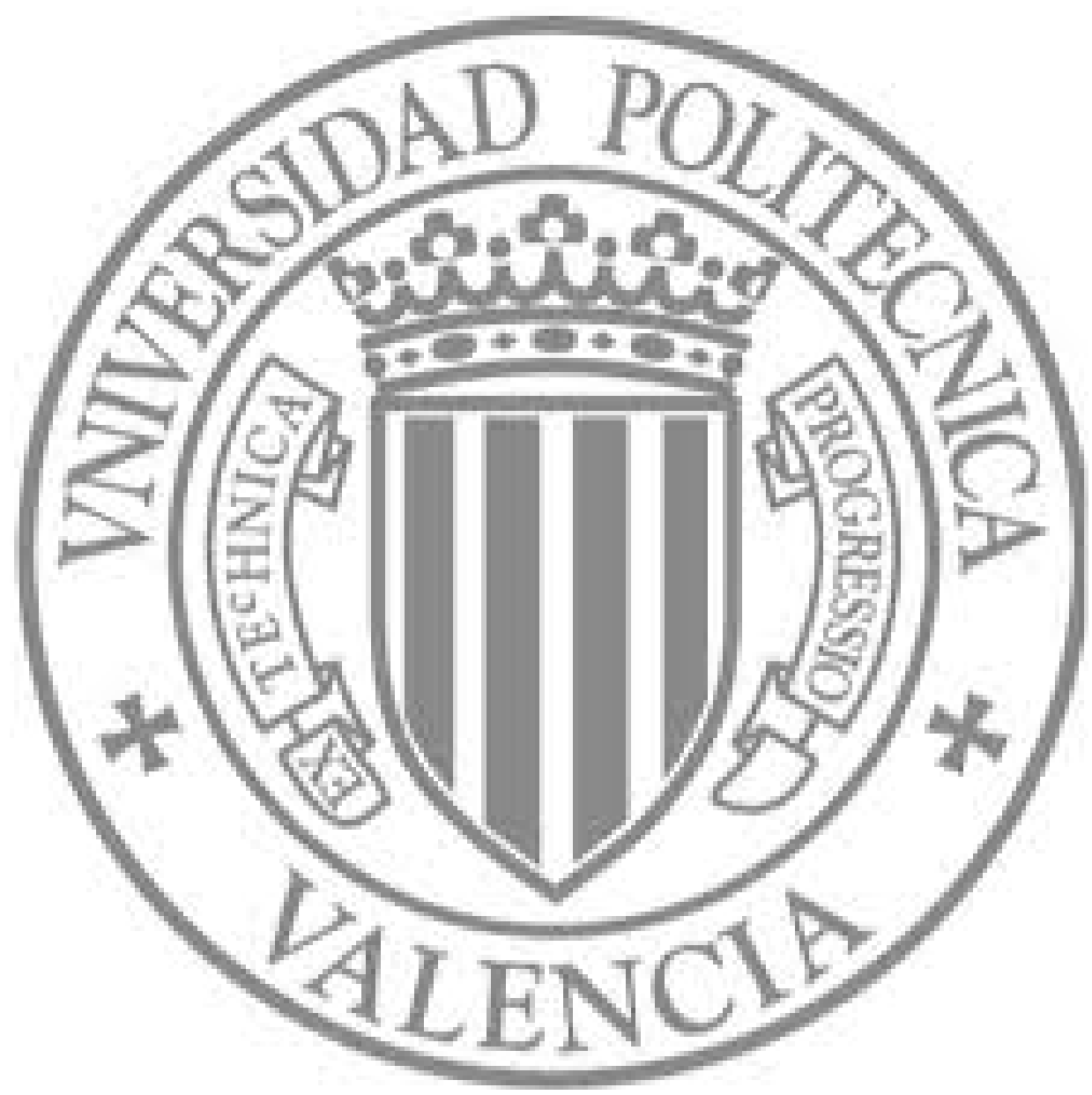

The final publication is available at

http://dx.doi.org/10.1016/j.jnoncrysol.2013.07.018

Copyright Elsevier 


\section{SILICA PHASE FORMED BY SOL-GEL REACTION IN THE NANO- AND MICRO-PORES OF A POLYMER HYDROGEL}

Journal of Non-Crystaline Solids 379 (2013) 12-20

http://dx.doi.org/10.1016/j.jnoncrysol.2013.07.018

Clara E. Plazas Bonilla ${ }^{\text {a,b, }}$ José A. Gómez-Tejedor ${ }^{\text {b }}$, Jairo E. Perilla ${ }^{c}$, José L. Gómez Ribelles ${ }^{\mathrm{b}, \mathrm{d}}$.

a. Universidad Nacional de Colombia - Sede Bogotá - Facultad de Ciencias - Departamento de Farmacia Grupo de Procesos Químicos y Bioquímicos - Av Cra 30 45-03, Bogotá, Código Postal 111321Colombia.

E-mail address: ceplazasb@unal.edu.co

b Center for Biomaterials and Tissue Engineering, Universitat Politècnica de València, Camino de Vera s/n, E-46022 Valencia, Spain.

c Universidad Nacional de Colombia - Sede Bogotá - Facultad de Ingeniería - Departamento de Ingeniería Química y Ambiental - Grupo de Procesos Químicos y Bioquímicos - Av Cra 30 45-03, Bogotá, Código Postal 111321- Colombia.

d CIBER en Bioingeniería Biomateriales y Nanomedicina (CIBER-BBN), Valencia, España.

Corresponding author José L. Gómez Ribelles .E-mail address: jlgomez@ter.upv.es

\section{Abstract}

Hybrid composites consisting in a hydrogel matrix with silica micro- and nano-particles reinforcement were produced and characterized. The strategy proposed in this work to obtain these composites consisted in a two-step synthesis, being the polymer network formation the first step. Porous poly(hydroxyethyl acrylate) polymer network was produced via free radical polymerization. Monomer and crosslinker were diluted in a varying amount of ethanol that controls the porosity of the resulting network. Polymeric microstructure drives the absorption of a silica precursor solution and the further distribution of the inorganic phase which was formed "in situ" in part occupying the pores and in part in the form of nanoparticles distributed in the polymer phase. Composites with silica content up to $60 \%$ by weight were obtained. In the case where the silica phase was continuous, samples maintained their integrity after eliminating the organic phase by pyrolysis. Water absorbed in the gel was able to crystallize, at least in 
part, when the silica content was below $30 \%$ by weight. Interestingly, for higher silica content the glass transition of the polymer phase was suppressed as well. Compliance was determined by indentation experiments. A continuous decrease in compliance was observed as filler content increased. Improvement of bioactivity of the material in simulated body fluid was also assessed. The synthetic route proposed allowed obtaining a family of composite hydrogels with variable properties.

\section{Highlights}

- A procedure to synthesize an interconnected silica nanophase in a previously formed polymer hydrogel.

- Silica nano- and micro-domains formed in a polymer nano-and micro-porous hydrogel by a sol-gel reaction.

- Hybrid materials with up to $60 \%$ by weight silica content in which both silica and organic phases are continuous.

Keywords: A. Hybrid composite, A. Nanocomposite, A. Polymer-matrix composites (PMCs), A. Particle-reinforced composites, E. Sol-gel methods.

\section{Introduction}

Important properties of cross-linked hydrogels that make them useful as a potential biomaterials are their remarkable ability to absorb water and their biocompatibility [13]. Swelling capacity of the hydrogels may be modulated by adjusting cross-linking density of the network and controlling porosity. A microporous hydrogel can be produced by diluting monomer and cross-linker in a suitable solvent since phase separation takes place during polymerization [4,5], being pore size and pore interconnectivity highly dependent on polymerization conditions, in particular the amount of cross-linker and the solvent content in the reacting mixture $[2,3,5,6]$. Due to their weakness when swollen, some studies have intended to improve their mechanical properties by different methods, for instance by copolymerization alternating hydrophilic and hydrophobic domains [7]. In this vein, the formation of organic/inorganic composites is a promising strategy for improving mechanical properties, as the advantages of the inorganic material (e.g., rigidity, thermal stability) are combined with those of organic polymers (e.g., flexibility, ductility, water sorption capacity, surface tension, processing). Composite properties are function of the strength 
of the interaction between the inorganic and inorganic phases at the interface as well as the amount of interface nanoparticles are expected to favor interface interaction $[8,9]$. Methods for obtaining polymer/silica nanocomposites include: (a) mixing silica nanoparticles and the polymer[10-14], (6) sol-gel processes in presence of a solution of the organic polymer $[10,13,15-18]$, and (c) in situ sol-gel process and simultaneous polymerization[12,14,19]. Introduction of functional groups along the backbone of the polymer can favor the formation of covalent bonds between organic and inorganic phases during sol-gel reaction $[9,17,20]$. All these methods have been widely studied to prepare hybrid hydrogel-silica nanocomposites. Polymer-silica hybrids have been obtained by using homopolymer and copolymer networks based in hydroxyethylmethacrylate (HEMA) and hydroxyethylacrylate (HEA) being polymerized simultaneously by sol-gel synthesis of the silica network [12-14,19,21, 22]. Polymerization of HEMA or HEA is slower than sol-gel reaction and thus, silica network formation takes place in presence of the monomer which acts as a template determining nano-porous structure of silica phase[23].

In previous studies poly(hydroxyethyl acrylate)(PHEA)-silica nanocomposites were produced by simultaneous polymerization of the two phases in a sol-gel process using tetraethylorthosilane (TEOS) as silica precursor. It was found that in composites with silica content above $15 \%$ by weight, organic and inorganic phases are co-continuous as probed by atomic force microscopy, nanoindentation and water sorption measurements [24,25]. Similar results were obtained in copolymer networks of HEA and ethyl acrylate (EA) $[26,27]$.

In this work, we explore a different way of producing PHEA-silica composites in which the first step is to produce the polymer network and then the solution containing silica precursor is absorbed in the bulk hydrogel using sol gel reaction to produce silica nanoparticles in the nanopores of the hydrogel structure. Higher silica contents can be obtained producing macro porous PHEA hydrogel. In this way, nanocomposites with silica content up to $60 \%$ by weight were obtained. When this gel is immersed in the precursor solution sol-gel reaction produces silica nanoparticles inside the polymer matrix as in the case of bulk hydrogel but, in addition silica is formed in micropores. The result is a double micro- and nanocomposite with silica content from $20 \%$ to $60 \%$ by weight. Bioactivity, some physical properties (as water and polymer mobility) and some thermal and mechanical properties were analyzed. 


\section{Materials and Methods}

2.1 Materials:

2-Hydroxyethylacrylate, (HEA, from Aldrich 96\%) was polymerized at room temperature under UV radiation for 6 hours using $0.5 \%$ wt of benzoin ( from Scharlau $98 \%$ ) as photo initiator and $1 \%$ of ethyleneglycol dimethylacrylate (EGDMA, from Aldrich, 98\%) as crosslinker to obtain bulk PHEA. In order to obtain porous PHEA films monomers were diluted in ethanol (from Scharlau, synthesis grade). The weight fraction of ethanol in the monomer mixture ranged between 0 and $50 \%$. The sheets produced, around $1 \mathrm{~mm}$ thick, were subjected to a post curing treatment at $90^{\circ}$ for 24 hours. Samples were washed with distilled water for 24 hours at approximately 60$70^{\circ} \mathrm{C}$, changing water every 8 hours, dried at room temperature for 24 hours and then in a vacuum desiccator for additional 24 hours. Samples will be designed PHEAXX, being XX the weight fraction of ethanol in the monomer solution.

Hybrid composites were prepared by sol-gel reaction using TEOS (from Aldrich, 98\%) as silica precursor and hydrochloric acid, $\mathrm{HCl}$ as catalyzer. Solution of silica precursor consisted in TEOS/water/ethanol/HCl mixtures with molar ratios 1/15/1/0.0185. TEOS was mixed with water and ethanol under continuous stirring for 5 minutes, then $\mathrm{HCl}$ was added and the solution was stirred 60 additional minutes. Porous or bulk PHEA were then immersed in this solution and allowed to swell at room temperature for 24 hours in order to attain constant weight and let to take place hydrolysis of silica precursor simultaneously. Sol gel reaction continued then at $40^{\circ} \mathrm{C}$ for another 24 hours. Finally the samples were washed in water/ethanol 15/1 mixtures and dried in vacuum at $40^{\circ} \mathrm{C}$ to constant weight. The samples were designated PHEAXXSI, where XX has the same meaning as above.

\subsection{Materials Characterization}

\subsubsection{Inorganic Hybrid composition}

Silica content of the hybrid composites was determined by weighing of the dry samples before and after sol-gel reactions. Residues after pyrolysis up to $850^{\circ} \mathrm{C}$ in oxygen atmosphere and residues after thermogravimetric analysis, TGA in nitrogen atmosphere (measured in a heating scan at $10^{\circ} \mathrm{C} / \mathrm{min}$ up to $850^{\circ} \mathrm{C}$ in a SDT Q600 analyzer, TA Instruments, United States) were also measured.

\subsubsection{Water Sorption}

Equilibrium water sorption was measured by immersion of pieces of materials in purified water at $37^{\circ} \mathrm{C}$. Sorption kinetics was measured by weighing at 1, 2, 8, 16 and 24 
hours (time at which all PHEA sample compositions attained an equilibrium plateau). Computed equilibrium water content EWC was calculated as the weight of water absorbed at 24 hours per gram of dry sample.

\subsubsection{Scanning Electronic Microscopy (SEM)}

A Scanning Electron Microscope Jeol JSM-5410 equipped with a cryounit Oxford CT 1500 using the low-temperature freeze drying technique (cryoSEM), was used to examine surface and cross-sections of samples which were swollen in water after $24 \mathrm{~h}$ in order to open the pores of hydrogels and composites. Water was sublimated at $-90{ }^{\circ} \mathrm{C}$ for 90 minutes. Samples were cryo-fractured inside the microscope, and sputtered with a gold layer. Micrographs were taken at an accelerating voltage of $20 \mathrm{kV}$ in order to ensure a suitable image resolution.

Homogeneity of silica phase distribution was assessed both at the surface and crosssection by Energy Dispersive X ray Spectroscopy, EDS (from Oxford Instruments), performed over the surface and over cross section samples. Samples were previously sputter-coated with carbon using an acceleration voltage of $10 \mathrm{KV}$ and a distance of 15 $\mathrm{mm}$. Silica was used as optimization standard.

\subsubsection{Atomic Force Microscopy (AFM)}

AFM, was performed in a NanoScope IIIa from Digital Instruments, operating in tapping mode in air. Si-cantilevers from Nanoworld were used with constant force of $2.8 \mathrm{~N} / \mathrm{m}$ and nominal resonance frequency of $92-95 \mathrm{kHz}$. The phase signal was set to zero at the resonance frequency of the tip. The tapping frequency was $5-10 \%$ lower than the resonance one. Drive amplitude was 3.0 -4.0 V and the amplitude set point 2.7$3.8 \mathrm{~V}$

\subsubsection{Fourier Transformed Infrared Spectroscopy (FTIR)}

FTIR spectra were obtained between 600 and $4000 \mathrm{~cm}^{-1}$ with a Nexus Spectrometer (Nicolet Instruments LTD, Warwick, UK) at room temperature using Attenuated Total Reflectance technique, ATR (in case of soft PHEA films), or Smart Diffuse Reflectance technique, SDR, (in case of the composites) depending on the sample. All the recorded spectra are the averages of 64 scans for each specimen. As a reference it was used silica gel 0.06-0.2mm from Scharlau.

\subsubsection{Differential Scanning Calorimetry, DSC}

DSC heating and cooling scans were performed in a Mettler Toledo 823e DSC at a scan rate of $10 \%$ min under a dry nitrogen atmosphere. The sample weights ranged between 5 
and $10 \mathrm{mg}$. Amount of crystallizable water in the samples was determined calibrating heat flow with the melting heat of pure water.

\subsubsection{Mechanical Test}

\subsubsection{Indentation Test}

Mechanical tests were performed at room temperature using dry samples (aprox.5mm $\mathrm{x}$ $6 \mathrm{~mm} \times 1 \mathrm{~mm}$ ) in a Seiko Extar TMA 7ss6000 (Seiko Instruments) in indentation mode. A $0.785 \mathrm{~mm}^{2}$ plane ended circular indenter and ramp forces at a rate of $0.005-0.05$ $\mathrm{N} / \mathrm{min}$ for PHEA films and $0.5-2.0 \mathrm{~N} / \mathrm{min}$ for hybrids were applied to reach penetration of at least $45 \mu \mathrm{m}$ in each of the samples. Poisson coefficient of the samples was determined from the shape of prismatic samples during a tensile experiment. Finite element simulation, assuming isotropous deformation of the cross section of the sample allowed to determine an average value of $v=0.4$ that was used in the calculation of the compliance from indentation results (see below)

\subsubsection{Dynamic Mechanical Analysis}

Dynamic mechanical measurement was performed in a DMS210 Seiko Analyzer in stretching mode, using a dry prismatic sample of $16^{*} 6^{*} 1.3 \mathrm{~mm}$ of PHEA0SI. Samples were scanned at $1 \mathrm{~Hz}$ in a heating rate at $1^{\circ} \mathrm{C} /$ minute between 25 to $120^{\circ} \mathrm{C}$ and after an isotherm of 20 minutes, a cooling scan at $1^{\circ} \mathrm{C} / \mathrm{min}$ to $25^{\circ} \mathrm{C}$.

\subsubsection{Bioactivity Assay}

Simulated Body Fluid (SBF) with the following ion concentration's was prepared: 142 $\mathrm{mM}$ of $\mathrm{Na}^{+}, 5.0 \mathrm{mM}$ of $\mathrm{K}^{+}, 2.5 \mathrm{mM}$ of $\mathrm{Ca}^{+2}, 1.5 \mathrm{mM}$ of $\mathrm{Mg}^{+2}, 148.8 \mathrm{mM}$ of Cl-, $4.2 \mathrm{mM}$ of $\mathrm{HCO}^{-}, 1.0 \mathrm{mM}$ of $\mathrm{HPO}^{-}$and 0.5 of $\mathrm{SO}^{-2}, \mathrm{pH}$ of 7,4 and temperature of $37^{\circ} \mathrm{C}$ [28].Dry weighted PHEA or composite pieces of around 6 × 6 x $1 \mathrm{~mm}$ were immersed in approximately $3 \mathrm{ml}$ of SBF suspended in the middle of the glass flask using a plastic thread and placed in an oven at $37^{\circ} \mathrm{C}$. The SBF solution was renewed the third 3 day. After 7 days, half of the samples were gently rinsed with purified water, let to dry first at room conditions for 24 hours and then in vacuum desiccators at room temperature. The immersion solution of remaining samples, was changed by a similar volume of $\mathrm{SBF}^{*} 2$ (containing double ions concentration), and let stand at the same temperature for an additional week. The $\mathrm{SBF}^{*} 2$ solution was renewed the 10th day. At day fourteen the samples were washed and dried as indicated above. Sample's final weight was made and, morphology (SEM) and composition of the apatite coating (EDS) were assessed.

\section{Results}


"In situ" absorption of a silica precursor solution and sol-gel reaction in the PHEA gel allowed obtaining nano-composites with silica content from around 20 to $60 \% \mathrm{w} / \mathrm{w}$. A series of samples were obtained ranging from soft hydrogels to high stiff and brittle materials whose water sorption capacity decreased with respect to the matrix polymer as silica content increased. All hybrid materials have a high transparency, a fact that can be understood as a previous reliable argument indicating that silica particle size is very small.

\subsection{Scanning Electron Microscopy (SEM)}

The images recorded using cryo SEM technique were in first stage used for verifying the microstructure of PHEA films and PHEA-silica composites. Figure 1a shows the pore structure of PHEA polymerized with $50 \%$ ethanol. The pore size observed, up to 5 microns approximately corresponds to the hydrogel swollen in liquid water. Nevertheless, cross-section of silica nanocomposites is smooth without any sign of pores (Figure 1b). To check the homogeneity of silica distribution in the whole sample, EDS spectra were recorded both at the surface and in cross-sections of the sample (see the inset in Figure 1b). The peaks corresponding to $\mathrm{Si}$ (in addition to oxygen) were observed in diverse points of the surface and cross section evidencing the homogeneity of the composite. The atomic ratio between oxygen and silicon was around 2 in most of the points analyzed although but in several points of the sample values between 0.5 and 3 were obtained.

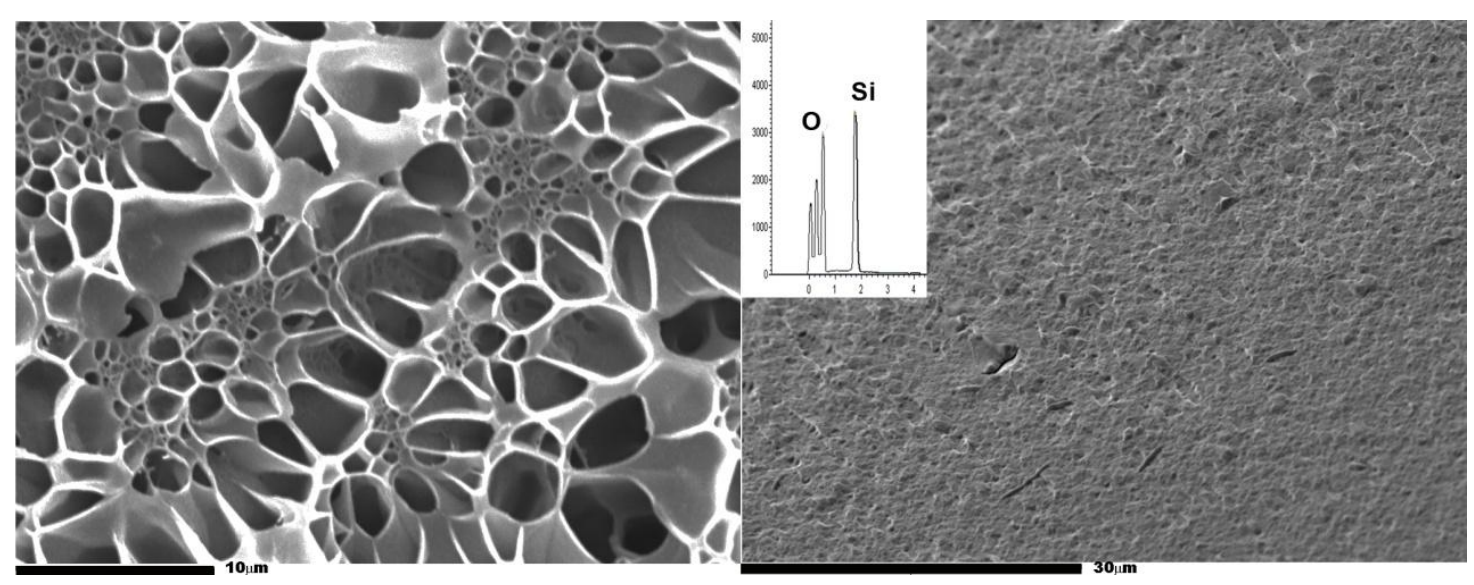

Figure 1. CryoSEM image of a) PHEA50 film b) PHEA50SI hybrid. In the inset a typical EDS analysis used to assess the homogeneous presence of silicon in PHEASILICA hybrids is shown 


\subsection{Atomic Force Microscopy (AFM).}

AFM was used to obtain information about silica distribution. Figure 2 presents AFM amplitude images of hybrid materials. Figure $2 \mathrm{a}$ shows the structure of the composite obtained by sol-gel reaction prepared from a bulk (non-porous) PHEA hydrogel, scanning an area of $1 \times 1 \mu \mathrm{m}$. Nanometric dispersed domains are clearly shown in the sample cross-sections. In the composites prepared from a porous PHEA hydrogel in addition to these nanometric silica domains, larger micrometric aggregates could be observed (arrows in Figure 2b), corresponding to hybrid prepared from the hydrogel with $50 \%$ ethanol scanned in a $10 \times 10 \mu \mathrm{m}$ area.

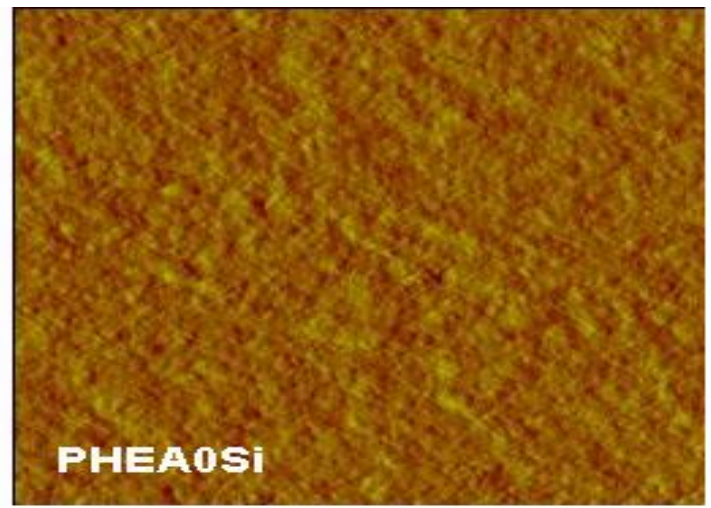

$2 \mu \mathrm{m}$

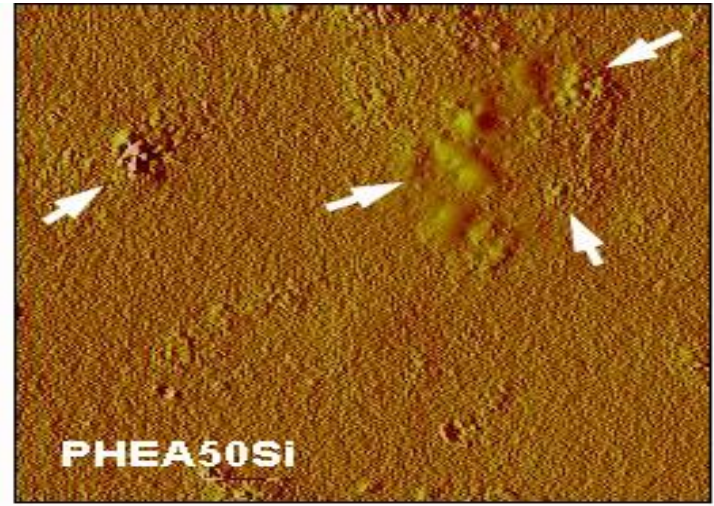

$10 \mu \mathrm{m}$

Figure 2. Amplitude AFM images of a) PHEA0SI and b) PHEA50SI hybrid composites. Arrows in PHEA50SI image shows the micrometric aggregates of silica particles produced in the macropores of PHEA50 hydrogel.

\subsection{Water sorption}

The equilibrium water content (EWC), defined as $\mathrm{g}$ water/g dry sample determined by difference in weighing after immersion of the samples in purified water for 24 hours (equilibrium time in agreement with previous PHEA hydrogels studies) [2,29]. As it is shown in Table 1 EWC grows rapidly with the amount of ethanol used in the polymerization due to the extra water that can accommodate in pores.

The swollen system is considered to consist of two phases, one formed by the polymer network with absorbed water occupying a volume, $V_{\text {swollen polymer }}$ The other phase is formed by pure liquid water occupying the volume of the pores $V_{\text {pores }}$. Thus, the volume fraction of pores in the swollen hydrogel can be calculated as:

$$
\phi_{\text {swollen }}=\frac{V_{\text {pores }}}{V_{\text {pores }}+V_{\text {swollen polymer }}}=\frac{v_{\text {water }}\left(\mathrm{w}-\mathrm{w}_{B}\right)}{v_{\text {swollen } B}\left(1+\mathrm{w}_{B}\right)+v_{\text {water }}\left(\mathrm{w}-\mathrm{w}_{B}\right)}
$$


where $v_{\text {water }}$ is the specific volume of pure water, $v_{\text {swollen } B}$ and $\mathrm{w}_{B}$ are the specific volume and the water uptake of the non-porous sample with the same crosslinking density respectively, and $\mathrm{w}$ is the water uptake of the porous sample after immersion. $v_{\text {swollen } B}$ was estimated assuming zero excess volume in the polymer/water blend, thus:

$$
v_{\text {swollen } B}=v_{\text {water }} \omega_{B}+v_{B}\left(1-\omega_{B}\right)
$$

where $v_{B}$ is the specific volume of the non-porous sample in the dry state and $\omega_{B}$ is the mass fraction of water in the sample in the swollen state, calculated as $\omega_{B}=\mathrm{w}_{B} /(1+$ $\mathrm{w}_{B}$ ). Then EWC can be used to estimate the volume fraction of pores in the swollen sample, using Equation (1) [5]. Values obtained are shown in Table 1.

The same procedure was followed to determine EWC for hybrid materials and as shown in Table 1, once silica is introduced in PHEA films, the ability of the hybrids to absorb water decrease dramatically.

Table 1. Some measured physical properties of PHEA films and PHEASILICA hybrids

\begin{tabular}{|c|c|c|c|c|c|c|c|c|}
\hline MATERIAL & $\begin{array}{l}\text { \% of Silica } \\
\text { content } \\
\text { (calculated } \\
\text { by } \\
\text { weighing) }\end{array}$ & $\begin{array}{l}\text { Residual } \\
\text { TGA } \\
\left(\mathrm{N}_{2}\right. \\
\text { atmosph } \\
\text { ere) }\end{array}$ & $\begin{array}{l}\text { Residual } \\
\text { pyrolysis } \\
\mathrm{CO}_{2} \\
\text { atmosphe } \\
\text { re) }\end{array}$ & $\begin{array}{l}\text { Porosity } \\
\text { of } \\
\text { swollen } \\
\text { sample } \\
\text { (\%) }\end{array}$ & $\begin{array}{l}\text { EWC/g } \\
\text { of sample } \\
\text { (\%dry } \\
\text { basis) }\end{array}$ & $\begin{array}{l}\text { EWC / g } \\
\text { of PHEA } \\
\text { (\%dry } \\
\text { basis) }\end{array}$ & $\begin{array}{l}\% \\
\text { of } \\
\text { crystalliza } \\
\text { ble water }\end{array}$ & $\begin{array}{l}\text { Creep Compliance } \\
(\mathrm{m} 2 / \mathrm{N})\end{array}$ \\
\hline PHEA0 & 0 & & & 0 & $190 \pm 7$ & $190 \pm 7$ & 88 & $\begin{array}{l}4.6 \mathrm{E}-06 \\
\pm 2.2 \mathrm{E}-07\end{array}$ \\
\hline PHEA20 & 0 & & & 24 & $274 \pm 8$ & $274 \pm 8$ & 87 & $\begin{array}{l}7.1 \mathrm{E}-06 \\
\pm 3.2 \mathrm{E}-07\end{array}$ \\
\hline PHEA30 & 0 & & & 40 & $369 \pm 14$ & $369 \pm 14$ & 83 & $\begin{array}{l}8.5 \mathrm{E}-06 \\
\pm 6.3 \mathrm{E}-07\end{array}$ \\
\hline PHEA40 & 0 & & & 51 & $474 \pm 25$ & $474 \pm 25$ & 91 & $\begin{array}{l}9.4 \mathrm{E}-06 \\
\pm 9.2 \mathrm{E}-07\end{array}$ \\
\hline PHEA50 & 0 & & & 69 & $794 \pm 33$ & $794 \pm 33$ & 89 & $\begin{array}{l}1.3 \mathrm{E}-05 \\
\pm 5.6 \mathrm{E}-07\end{array}$ \\
\hline PHEA0SI & 21.7 & 18.8 & 19.6 & - & $86 \pm 3$ & 109 & 79 & $\begin{array}{l}1.4 \mathrm{E}-06 \\
\pm 4.9 \mathrm{E}-07\end{array}$ \\
\hline PHEA20SI & 32.6 & 31.6 & 30.6 & - & $47 \pm 8$ & 70 & 66 & $\begin{array}{l}7.7 \mathrm{E}-07 \\
\pm 4.0 \mathrm{E}-08\end{array}$ \\
\hline PHEA30SI & 41.1 & 42.5 & 38.1 & - & $27 \pm 1$ & 46 & - & $\begin{array}{l}1.9 \mathrm{E}-07 \\
\pm 2.0 \mathrm{E}-07\end{array}$ \\
\hline PHEA40SI & 48.9 & 45.6 & 44.8 & - & $19 \pm 2$ & 37 & - & $\begin{array}{l}1.9 \mathrm{E}-07 \\
\pm 3.2 \mathrm{E}-08\end{array}$ \\
\hline
\end{tabular}




\begin{tabular}{|l|l|l|l|l|l|l|l|l|}
\hline PHEA50SI & 58.8 & 54.5 & 52.4 & - & $12 \pm 4$ & 30 & - & - \\
\hline
\end{tabular}

EWC: Equilibrium Water Content

\subsection{Weight fraction of silica phase in the composite.}

The amounts of silica phase in the composite determined by weighing before and after precursor absorption and sol-gel reaction are listed in Table 1. The rapid increase in silica content with the increase of the porosity of PHEA network is evident, reaching $\sim 59 \%$ by weight.

The sol-gel conditions selected for "in situ" hydrolysis and condensation of TEOS do not yield to full conversion of orthosilicate groups into silica, and thus the silica phase is expected to contain a certain amount of organic unreacted groups. This is revealed by measuring the weight residue after TGA (performed in nitrogen atmosphere) or pyrolysis (which is performed in oxygen atmosphere and thus yield a complete decomposition of organic components) being smaller than the fraction of inorganic phase calculated by weighing after sol-gel reaction. This conclusion is supported by the fact that the elastic modulus of the sample, measured in a DMTA heating scan, grows continuously when the temperature increases above $40^{\circ} \mathrm{C}$ indicating the progress of silica condensation at higher temperatures than those used to produce hybrids (see Figure 3). Modulus increase is irreversible as shown in the cooling scan. On the other hand, pyrolysis residue was a consistent method for confirming the homogeneous distribution of silica phase inside the polymer because hybrid samples submitted to the thermal process retained the original sample shape, being dissimilar only in their translucent appearance (Figure 4).

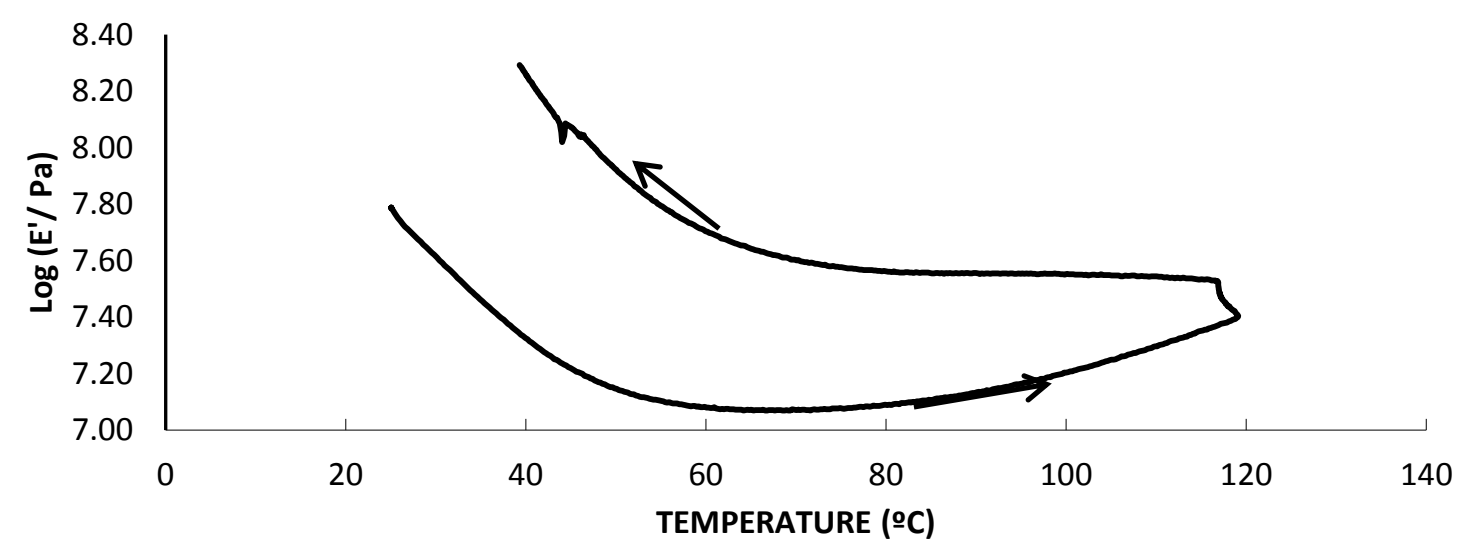


Figure 3. Temperature dependence of storage modulus ( E') of PHEA0SI sample subjected to a heating scan at $1 \mathrm{~Hz}$ between 25 and $120^{\circ} \mathrm{C}$ followed by cooling from 120 to $25^{\circ} \mathrm{C}$.

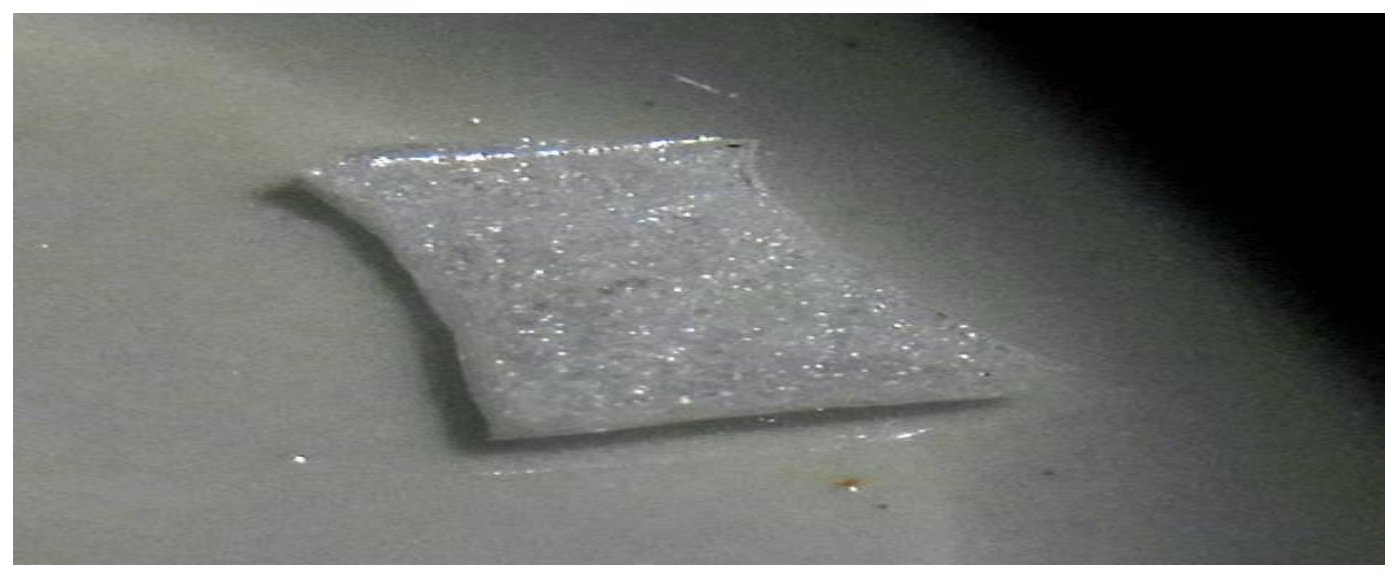

Figure 4. Macroscopic appearance of the pyrolysis PHEA50SI residue.

\subsection{Infrared spectroscopy}

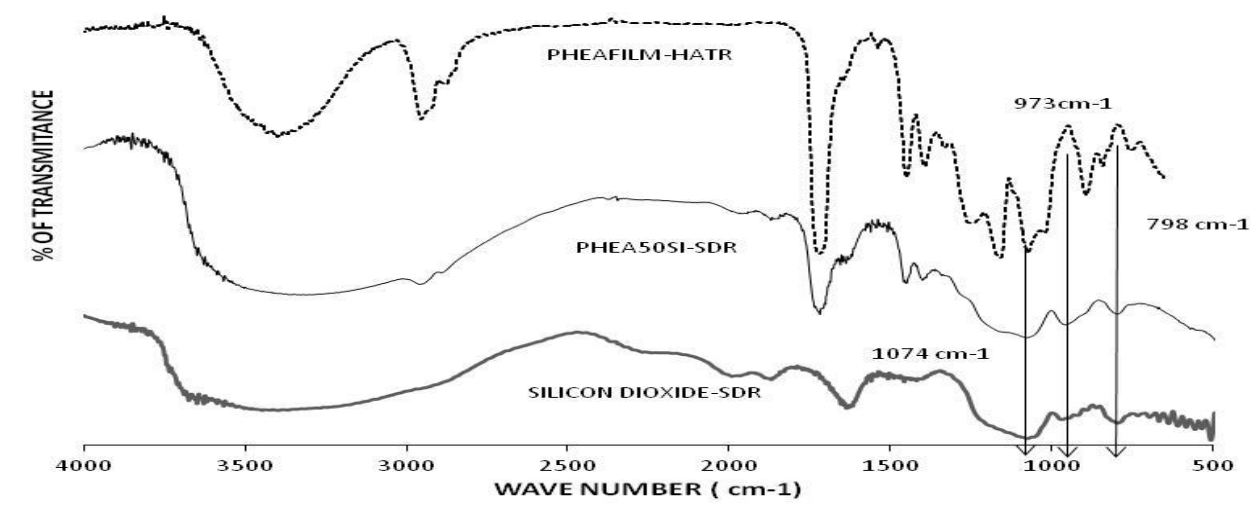

Figure 5. FTIR spectra of PHEA film and PHEA50SI composite. The spectra were shifted in the Y axis for the sake of clarity.

The FT-IR spectrum of PHEA network, (Figure 5), is in agreement with previous available results for the PHEA films [30]. A characteristic carbonyl peak $(\mathrm{C}=\mathrm{O}$ stretching) appears at $1710-1720 \mathrm{~cm}^{-1}$,Peaks in the range of $2900-3000 \mathrm{~cm}^{-1}$ correspond to the aliphatic $\mathrm{C}-\mathrm{H}$ stretching, the peak at $1410-1450 \mathrm{~cm}^{-1}$ is due to $-\mathrm{CH}_{2}$; and the peak at $3415 \mathrm{~cm}^{-1}$ corresponds to $\mathrm{O}-\mathrm{H}$ stretching. Furthermore, $\mathrm{O}-\mathrm{H}$ bending is observed at 
$1298 \mathrm{~cm}^{-1}$ and ester peak (C-O stretching) can be seen at $1200 \mathrm{~cm}^{-1}$. The broad peak at around $3500 \mathrm{~cm}^{-1}$ corresponds to $\mathrm{O}-\mathrm{H}$ bond stretching and also $\mathrm{O}-\mathrm{H}$ bending can be seen at around $1390 \mathrm{~cm}^{-1 .}$ The ester peak of the polymer is observed at $1160-1180 \mathrm{~cm}^{-1}$

As shown in Figure 5, in PHEASI nanocomposites spectrum presents the characteristic carbonyl peak at $1710-1720 \mathrm{~cm}^{-1}$.Peak at around $3500 \mathrm{~cm}^{-1}$ corresponding to $\mathrm{O}-\mathrm{H}$ bond tend to decrease with increasing silica content in the samples (results not shown). The bands at $973 \mathrm{~cm}^{-1}$ and $798 \mathrm{~cm}^{-1}$ are ascribed to bonds Si-OH and that at $1074 \mathrm{~cm}-1$ to $\mathrm{Si}-\mathrm{O}-\mathrm{Si}$. The spectrum of a silicon dioxide standard is also shown for comparison.

\subsection{Thermal Behavior}

The glass transition of the hybrid materials was only noticeable up to $20 \%$ wt silica content (results not shown). In the hybrid materials PHEA0SI and PHEA20SI it covers a broader temperature interval than in PHEA films and shifts towards higher temperatures as shown in the DSC thermograms of the inset of Figure 6. On the other hand, the heat capacity in the glass transition normalized by the weight fraction of polymer, $\Delta \mathrm{c}_{\mathrm{p}}\left(\mathrm{T}_{\mathrm{g}}\right) / \mathrm{W}_{\text {PHEA }}$, is $0.42 \pm 0.01$ for bulk and porous PHEA, while decreases until 0.39 for PHEA0SI and to 0.3 in PHEA20SI.

In the DSC heating thermograms recorded in PHEA wet samples (Figure 6), the melting peak of water is quite apparent in all PHEA samples. The onset of the peak is at $0^{\circ} \mathrm{C}$, although the thermogram starts deviating from the base line several degrees before. The area of the endotherm grows with the porosity of the sample as expected due to the increasing amount of water filling the pores. The average fraction of crystallizable water calculated from the melting heat and the water content of the PHEA samples was $87.6 \pm 2.3$ (obtained from data in Table 1) being independent of porosity. In the hybrid composites, the behavior with respect to water crystallization and melting is in some way parallel to that of the glass transition of the dry polymer phase: Sample PHEA0SI presents a fraction of crystallizable water that is similar to that of pure PHEA samples. In PHEA20SI a significant fraction of the water content is still able to crystallize $(66 \%)$ but it is smaller than in pure PHEA. A small melting peak is detected at $-20^{\circ} \mathrm{C}$ in PHEA30SI and no melting or crystallization is observed for higher silica content. Interestingly, the heating thermogram of PHEA20SI shows a first endotherm below $0^{\circ} \mathrm{C}$, which is followed by a sharp peak with onset a $0^{\circ} \mathrm{C}$. The thermogram of PHEAOSI 
is similar but the low-temperature peak appears as a shoulder of the high-temperature one (Figure 5).

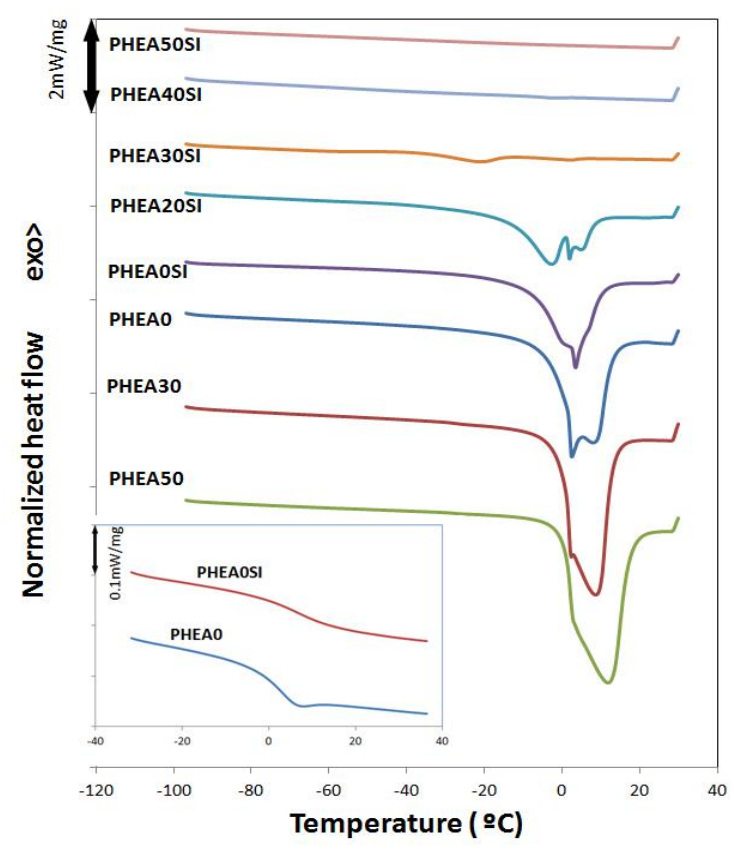

Figure 6. DSC heating thermograms of wet bulk and some porous PHEA and wet PHEASILICA hybrids composites. Inset: DSC heating thermograms of dry PHEA0 and PHEA0SI.

\subsection{Mechanical Properties.}

\section{Indentation Test}

Indentation test, with a cylindrical shape plane-ended indenter with a contact surface much smaller than the sample, allows characterizing the mechanical behavior of the material through the measured relationship between the applied force and the indenter penetration. As in other mechanical testing configuration if a controlled deformation profile is applied, what can be called stress indentation test (usually penetration at constant rate) the value of the Elastic Modulus of the material could be computed. In this case, a controlled time dependent force is applied, which can be called creep indentation test (a linearly increasing compression force is applied to the indenter) and in this way the experimental force-penetration curve allows calculating the material Compliance[31,32]. For the indentation of a plate with thickness $h$ of an elastic material with a stiff plane-ended cylindrical indenter with radius $a$, Hayes et. al. found the solution for the load ( $P$ )-penetration ( $W$ ) relationship as presented in equation 3 .

$$
P=\frac{4 a k}{(1-v)} G W
$$


where $k$ is a non dimensional parameter which depends on the ratio $a / h$ and the Poisson coefficient $v$ of the material [33] and $G$ is the elastic shear modulus.

The response of a linear viscoelastic solid to a creep indentation of any load history can be determined following the technique proposed by Lee and Radok[34]. This method was used by Wang et. al. [31] to find the solution of the load-penetration curve in the case of a conical or a spherical indenter. Following their method and using Hayes equation (3) for a plane-ended cylindrical indenter [33], it can be found

$$
J(t)=\frac{4 a k}{(1-v)\left[\frac{d P}{d t}\right]} \frac{d w}{d t}
$$

The values of $J(t)$ were evaluated at time that corresponds to a penetration $45 \mu \mathrm{m}$ in the sample. The results are shown in Table 1.

\subsection{Bioactivity Assay}

The bioactivity assay evaluated by SEM images of the samples surface after one week immersion in SBF did not show any clear deposition of apatite, nevertheless after two weeks apatite crystals with cauliflower morphology can be observed both in pure PHEA and in the composites, with a clear increment of apatite deposition in the composites with respect to pure PHEA samples. Figure 7 shows comparative SEM images for PHEA30 and PHEA30SI. Elemental analysis by EDS shows that the ratio calcium/phosphorous was higher for PHEASILICA samples (between 1.6 and 2.2) comparing to PHEA pure ( 1.4). The sample weight of pure PHEA sample increased by immersion in SBF due to apatite deposition. However the weight of the composites decreased due to the loss of silica solved in the SBF medium, being nearly independent of silica content in hybrids with silica content below $45 \%$ but higher for those with a higher amount of silica. This interpretation is further confirmed by an important increase of the ratio oxygen/silicon measured by EDS (Table 2). 


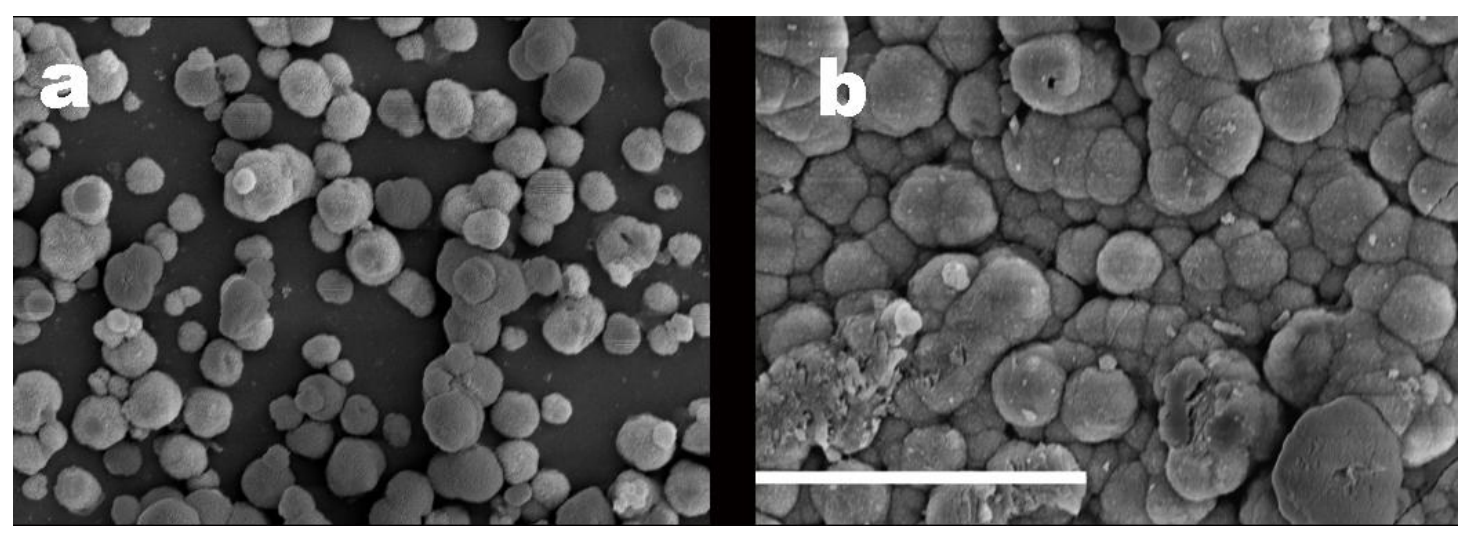

Figure 7. SEM microphotographs of PHEA30 (a) and PHEA30SI (b) after two weeks immersion in SBF. Dimension bar corresponds to $20 \mu \mathrm{m}$.

Table 2. Bioactivity results after two weeks immersion in SBF.

\begin{tabular}{|l|l|l|l|}
\hline MATERIAL & $\begin{array}{l}\text { CHANGE OF } \\
\text { WEIGHT( \%) }\end{array}$ & $\begin{array}{l}\text { AVERAGE FINAL } \\
\text { ATOMIC RATIO O/Si }\end{array}$ & $\begin{array}{l}\text { AVERAGE FINAL } \\
\text { ATOMIC RATIO Ca/P }\end{array}$ \\
\hline PHEAOSI & -5.0 & 31.2 & 2.2 \\
\hline PHEA20SI & -4.3 & 30.5 & 1.6 \\
\hline PHEA30SI & -4.5 & 79.8 & 2.2 \\
\hline PHEA40SI & -10.5 & & 1.4 \\
\hline PHEA50SI & -15.5 & & \\
\hline PHEA CONTROL & 3.2 & & \\
\hline
\end{tabular}

\section{Discussion}

Bulk PHEA, prepared by radical polymerization with 1\% EGDMA as cross-linker, is a polymer network with an equilibrium water uptake of $190 \%$ measured on dry basis. The distribution of water in the swollen polymer has been extensively studied in the past using calorimetry and dielectric spectroscopy [12,25], showing that a first layer of water molecules is bonded to adsorption sites of the polymer chains, but successive adsorption layers form water clusters with nanometric size in which water preserves its physical properties, including the ability to crystallize and melt. To form the hybrid composite, the polymer hydrogel was used as a template for the synthesis of silica phase through the absorption of a solution of silica precursor into the polymer matrix, and it was expected a uniform distribution of silica in nanometric scale, in regions as water does. 
In fact when sol-gel reaction takes place silica aggregates are uniformly distributed in the polymer phase as can be observed in AFM pictures (Figure 2a). The composite finally contains $22 \% \mathrm{wt}$ of the inorganic phase. Formation of silica was probed by FTIR (Figure 5) and EDS analysis (Figure 1). The reaction conditions used do not yield full conversion, as proved by the irreversible increase of the elastic modulus of the sample on further heating (Figure 3). On the other hand the weight of the composite sample after reaction allows determine that $22 \%$ corresponds to the inorganic phase while the residue after thermal degradation in nitrogen atmosphere in TGA or in oxygen atmosphere in the pyrolysis experiment is slightly smaller, what can be ascribed to the advance of condensation reaction and lost of unreacted ethyl groups. The composite thus obtained, can be considered a hydrogel, with a water sorption capacity around of $86 \%$ wt measured on dry basis. This means that the polymer phase is still able to swell absorbing important amounts of water, what indicates that silica nanoparticles are dispersed in the polymer phase and did not percolate to form a continuous silica network that would be unable to swell. Indentation experiments also supports this conclusion since creep compliance of the sample decreases from $4.610^{-6}$ to $1.410^{-6}$ $\mathrm{m}^{2} / \mathrm{N}$, but the difference is moderate because silica nanoparticles do not join to each other. DSC thermograms in the region of the glass transition of PHEA reflect the close packing of silica nanoparticles and polymer chains; glass transition is shifted towards higher temperatures and broadens. Glass transition is ascribed to the cooperative rearrangements of the polymer chains in regions with some nanometers or tens of nanometers size $[35,36]$. The fact that the temperature interval in which the transition takes place becomes broader means that the cooperative rearranging regions become more heterogeneous because of the presence of silica nanoparticles. But interestingly the onset of the transition is shifted towards higher temperatures. It may be interpreted in the sense that no rearranging region behaves as in the pure polymer and so all the polymer chains have silica particles at distances in the order of several nanometers what speak about the homogeneous distribution of silica in the composite. As a consequence, it could come to conclude that the formulation used for the silica precursor is homogeneously absorbed by PHEA. In the swollen composite sample water is still able to crystallize as can be seen by the melting peaks shown by the heating thermogram of Figure 6. When the melting peak of the composite is compared with that in pure PHEA swollen network, the differences are quite clear. In PHEA, the onset of melting is situated at $0^{\circ} \mathrm{C}$, as corresponds to a water phase separated of the polymer chains. 
Nevertheless, in the composite the thermogram starts deviating from the baseline in the endothermic direction around $-40^{\circ} \mathrm{C}$, producing a broad endotherm on which another endotherm, very sharp, overlaps at $0^{\circ} \mathrm{C}$. During water crystallization, on cooling, the part of the absorbed water that is distributed in separated water domains already in the liquid phase form solid structures large enough to melt as pure water. Nevertheless, the homogeneous liquid polymer-water mixture on cooling forms two separated phases: a polymer glass and water crystals. Melting of these structures initiate at lower temperatures, as it is shown in Figure 6. The different melting behavior in PHEA and in silica composite shows the different capacity of water to migrate from the polymer phase to incorporate to the large water crystals.

Hybrid composites can be obtained with this procedure starting with a hydrogel with increased porosity. Growth of the polymer network in the polymerization of hydroxyethyl acrylate diluted with a certain amount of ethanol yields a phase separation between the growing network and a liquid phase containing ethanol and the unreacted monomer. At the end of the reaction, the sample is washed in water which substitutes ethanol swelling the polymer and filling the pores. The huge increase of water content in the samples equilibrated in liquid water demonstrates that the sample is highly porous. In fact water uptake allows calculating the volume fraction of pores. As Table 1 shows the addition of up to $50 \%$ ethanol to the monomer in the polymerization allows obtaining hydrogels with equilibrium water content up to $800 \%$ wt measured on dry basis, in which the pores occupy a $69 \%$ of the sample volume. It is important to note that if the sample is dried the pore structure collapses and the apparent density of the polymer is that of non-porous material. Pore collapse is reversible by immersion in liquid water reopening pores and attaining original values of water uptake. Nevertheless, the properties of the xerogels, depends on the original porosity when swollen. When pore collapses leaves inside the sample discontinuity surfaces that make the material more deformable than it would be expected, as Table 1 shows, the creep compliance of dry samples polymerized with different amounts of ethanol increases linearly with the amount of diluent added in the polymerization, explained by the increase of pore size with the amount of diluent. That fact was observed by cryo-SEM, where samples immersed in water were frozen and observed after water sublimation in SEM. Thus, the pore is observed as it is in the swollen sample. Figure 1 show the microphotograph obtained in the sample with the highest porosity, giving evidence that pore structure consists in interconnected pores with micrometric dimensions. 
When porous hydrogels are swollen in silica precursor, it is absorbed in part by the polymer and in part filling the macropores, the sol-gel reaction forms dispersed silica nanoparticles distributed in the polymer phase and nano or micro-particles inside the macropores. When the sample is dried, pores collapse and the silica particles that were inside them agglomerate, as can be observed in the AFM pictures of Figure 2b. Arrows in this picture indicate the silica aggregates. In this way, the structure of the hybrid composite is that of a double nano- and micro-reinforced hydrogel. It can be said that it consists of a phase similar to PHEA0SI composite (we will call it the PHEASI phase) and another one consisting of silica micrometric aggregates. The amount of silica in the composite can be increased up to $60 \%$ wt. Water sorption capacity decrease monotonously with increasing silica content as expected because water is absorbed in the polymer phase, but it is worth note that the amount of water per gram of PHEA decreases with total silica content as well. Interestingly the water content per gram of polymer in the porous composite goes below that of PHEA0SI, what means that the PHEASI phase is not able to absorb the same amount of water as the pristine polymer. Furthermore, about $79 \%$ of absorbed water in PHEA0SI is able to crystallize, a fraction that decreases to $66 \%$ in PHEA20SI, while in composites prepared from more porous hydrogels freezing or melting of water cannot be detected by DSC. These results supported that pore connectivity in the original hydrogel produces a continuous inorganic phase that hinders swelling of the PHEASI phase. Continuity of silica phase is still confirmed by the coherence of the sample after the polymer phase is eliminated by pyrolysis (Figure 4). In addition the small values of creep compliance of dry sample further support that silica percolates and impedes polymer deformation. As Cryo-SEM images show, the hybrid composites obtained from porous hydrogels are no longer porous or at least pores do not reopen when samples are immersed in liquid water (Figure 1). Bioactivity assessment according to Kokubo's method shows significant differences in the microscopic appearance of the surfaces of PHEA hydrogels and the composites after two weeks immersion in SBF. EDS analysis and SEM images indicate the formation of a calcium phosphate layer on the surface and at the same time the loss of silica which is dissolved in SBF. The weight loss of the sample (not observed in pure PHEA hydrogels) must be due to silica dissolution.

\section{Conclusions}


PHEA hydrogel can be swollen with a silica precursor solution and by a sol-gel reaction a nanocomposite can be produced that in spite of containing around $20 \%$ wt silica it is compliant and able to absorb up to $80 \%$ wt of water measured on dry basis. Silica content of the composite can be increased up to $60 \%$ wt by absorption over a porous hydrogel. Then a two phase structure results, one of them consisting of a PHEA/silica nanocomposite similar to that formed from a non-porous hydrogel, while the second one consists of interconnected silica agglomerates. The continuous silica phase is responsible for the high stiffness of the composite and hinders water sorption since impedes swelling of the polymer phase. Then a broad range of hydrogel composites with varying mechanical and water sorption capacities can be produced by the method proposed in this work. The possibility of forming the inorganic phase in an already shaped hydrogel opens many possibilities of obtaining materials formed by alternating soft and hard phases.

\section{Acknowledgements}

CEPB acknowledges the economical support of COOPEN agreement in the progress of the present work. JLGR acknowledges the support of the Spanish Ministry of Education through project No. MAT2010-21611-C03-01 (including the FEDER financial support). The support of the Instituto de Salud Carlos III (ISCIII) through the CIBER initiative of the Networking Research Center on Bioengineering, Biomaterials and Nanomedicine $(C I B E R-B B N)$ is also acknowledged. Authors want to thank the technical support of the Universitat Politècnica de València’s Microscopy Service.

\section{References}

1. Hoffman AS. Hydrogels for biomedical applications. Adv Drug Deliv Rev 2002;54(1):3-12.

2. Monleón Pradas M, Gómez Ribelles JL, Serrano Aroca A, Gallego Ferrer G, Suay Anton J, Pissis P. Interaction between water and polymer chains in poly(hydroxy ethylacrylate) hydrogels. Colloid Polymer Science 2001;279:323-330.

3. Pal K, Banthia AK, Majumdar DK. Polymeric hydrogel: Characterization and Biomedical Applications : A mini review. 2009; 12:197-220.

4. Castilla Cortazar I, Vidaurre A, Gallego Ferrer G, Monleón Pradas M, Gómez Ribelles JL, Meseguer Dueñas JM. Hydrophilic sponges based on poly(hydroxyethy lacrylate). Journal of Non Cristalline Solids 2001;287:130-134.

5. Serrano Aroca A, Campillo Fernández A, Gómez Ribelles JL, Monleón Pradas M, Gallego Ferrer G, Pissis P. Porous poly(2hydroxyethyl acrylate) hydrogels prepared by radical polymerisation with methanol as diluent. Polymer 2004;45:8949-8955.

6. Serrano Aroca A, Monleón Pradas M, Gómez Ribelles JL. Macroporous poly(methyl methacrylate) produced by phase separation during polymerisation in solution. Colloid Polymer Science 2007;285:753-760. 
7. Salmerón Sánchez M, Brígido Diego R, Iannazzo SAM, Gómez Ribelles JL, Monleón Pradas M. The structure of poly(ethyl acrylate-co-hydroxyethyl methacrylate) copolymer networks by segmental dynamics studies based on structural relaxation experiments. Polymer 2004;45(7):2349-2355

8. Zou H, Wu S, Shen J. Polymer/Silica Nanocomposites: Preparation , Characterization, Properties and Applications. Chemical Reviews 2008;108(9):3893-3957.

9. Valliant EM, Jones JR. Softening bioactive glass for bone regeneration: sol-gel hybrid materials. Soft Matter 2011;7:5083-5095.

10. Boisvert JP, Persello J, Guyard A. Influence of the surface chemistry on the structural and mechanical properties of silica polymer composites. Journal of Polymer Science Part B: Polymer Physics 2003.;41:3127-3138.

11. Sirousazar M, Kokabi M, Hassan ZM. Swelling behavior and structural characteristics of Polyvinyl Alcohol/Montmorillonite nanocomposite hydrogels. Journal of Applied Polymer Science 2012;123:50-58.

12. Costantini A, Luciani G, Annunziata G, Silvestri B, Branda F. Swelling properties and bioactivity of silica gel/pHEMA nanocomposites. Journal of Materials Science: Materials in Medicine 2006;17(4):319-325.

13. Huang S, Chin W, Yang WP. Structural characteristics and properties of silica/poly(2-hydroxyethyl methacrylate) (PHEMA) nanocomposites prepared by mixing colloidal silica or tetraethyloxysilane (TEOS) with PHEMA. Polymer 2005;46(6):1865-1877.

14. Liu Y, Liu T, Chen S, Liu D. Synthesis and characterization of nanoporous $\mathrm{SiO}_{2} / \mathrm{pHEMA}$ biocomposites. Journal of Materials Science: Materials in Medicine 2008;19(8):2903-2911.

15. Pereira M, Jones J, Orefice L, Hench L. Preparation of biactive glass-polyvinyl alcohol hybrid foams by sol-gel method. Journal of Material Science : Material Science in Medicine 2005;16:1045-1050.

16. Liu W, Zhu B, Zhang J, Xu Y. Preparation and dielectric properties of polyimide/silica nanocomposite films prepared from solgel and blending process. Polymers for Advanced Technologies 2007;18:522-528.

17. Poologasundarampillai G, Ionescu C, Tsigkou O, Murugesan M, Hill RG, Stevens M, Hanna JV, Smith ME, Jones JR. Synthesis of bioactive class II poly([gamma]-glutamic acid)/silica hybrids for bone regeneration. Journal of Materials Chemistry 2010;20(40):8952- 8961.

18. Reis EM, Vasconcelos WL, Mansur HS, Pereira MM. Synthesis and characterization of silica -chitosan porous hybrids for Tissue Engineering. Key Engineering Materials 2008;361-363:967-970.

19. Hajji P, David L, Gerard JF, Pascault JP, Vigier G. Synthesis, structure, and morphology of polymer-silica hybrid nanocomposites based on hydroxyethyl methacrylate. Journal of Polymer Science Part B: Polymer Physics 1999;37(22):3172-3187.

20. Baino F, Vitale-Brovarone C. Three-dimensional glass-derived scaffolds for bone tissue engineering: Current trends and forecasts for the future Review. Journal of Biomedical Materials Research A, 2011;97A(4):514-535.

21. Kakwere H, Perrier S. Design of complex polymeric architectures and nanostructured materials/hybrids by living radical polymerization of hydroxylated monomers. Polymer Chemistry 2011;2:270-288.

22. Lin D, Chen C, Chang C, Su Y, Cheng L. Observation of Nano-Particles in Silica/poly(HEMA) Hybrid by Electron Microscopy Journal of Polymer Research 2002;9:115-118

23. Rodríguez-Hernández JC, Monleón Pradas M, Gómez Ribelles JL. Properties of poly(2-hydroxyethyl acrylate)-silica nanocomposites obtained by sol-gel process. Journal of Non-Crystalline Solids 2008;354(17):1900-1908.

24. Rodríguez Hernández JC, Salmerón Sánchez M, Gómez Ribelles JL, Monleón Pradas M. Polymer-silica nanocomposites prepared by sol-gel technique: Nanoindentation and tapping mode AFM studies. European Polymer Journal 2007;43(7):2775-2783.

25. Pandis C, Spanoudaki A, Kyritsis A, Pissis P, Rodríguez Hernández JC, Gómez Ribelles JL, Monleón Pradas M. Water sorption characteristics of poly(2-hydroxyethyl acrylate)/silica nanocomposite hydrogels. Journal of Polymer Science Part B: Polymer Physics 2011;49(9):657-668.

26. Stathopoulos A, Klonos P, Kyritsis P, Pissis P, Christodoulides C, Rodriguez Hernández J, Monleón Pradas M, Gómez Ribelles JL. Water sorption and polymer dynamics in hybrid poly(2-hydroxyethyl-co-ethyl acrylate)/silica hydrogels European Polymer Journal 2010;46:101-111.

27. Vallés-Lluch A, Rodríguez-Hernández JC, Ferrer GG, Pradas MM. Synthesis and characterization of poly(EMA-co-HEA)/SiO2 nanohybrids. European Polymer Journal 2010;46(7):1446-1455. 
Clara E. Plazas Bonilla, Journal of Non-Crystaline Solids 379 (2013) 12-20

28. Vallés Lluch A. P(EMA co HEA) / SiO2 hybrid nanocomposites for guided dentine tissue regeneration : Structure, characterization and bioactivity., Valencia España, Universidad Politécnica de Valencia, 2008.

29. Monleón Pradas M, Gómez Ribelles JL, Serrano Aroca A, Gallego Ferrer G, Suay Antón J, Pissis P. Porous poly(2hydroxyethyl acrylate) hydrogels . Polymer 2001;42:4667-4674.

30. Vargün E. Polymerization and characterization of 2-hydroxyethyl acrylate., Ankara Turkish, The Middle East Technical University, 2003

31. Lu H, Wang B, Ma J, Huang G, Viswanathan H.

Measurement of Creep Compliance of Solid Polymers by Nanoindentation. Mechanics of Time-Dependent Materials 2003;7:189207

32. Mak AF, Lai WM, Mow VC. Biphasic indentation of articular cartilage I. Theoretical analysis. J Biomechanics, 1987;20(7):703714

33. Hayes WC, Keer LM, Herrmann G, Mockros LF. A Mathematical analysis for indentation test of articular cartilage. J Biomechanics 1972;5:541-551.

34. Lee EH, Radok JRM.

The contact problem for viscoelastic bodies. Journal of Applied Mechanics 1960;27:438-444.

35. Gómez Ribelles JL, Vidaurre Garayo A, Cowie JMG, Ferguson R, Harris S, McEwen IJ. The length of cooperativity at the glass transition in poly(vinyl acetate) from the modelling of the structural relaxation process. Polymer 1998;40:183-192.

36. Donth E, Beiner M, Reissing S, Korus J, Garwe F, Vieweg S, Kahle S, Hempel E, Schröter K. Fine structure of the main transition in amorphous polymers: Entanglement, spacing and characteristic length of the glass transition. Discussion of Examples. Macromolecules 1996;29(20):6589-6600. 